\title{
Existence Results for Fuzzy Partial Differential Inclusions
}

\author{
Nayyar Mehmood ${ }^{1}$ and Akbar Azam ${ }^{2}$ \\ ${ }^{1}$ Department of Mathematics and Statistics, International Islamic University, H-10, Islamabad 44000, Pakistan \\ ${ }^{2}$ Department of Mathematics, COMSATS Institute of Information Technology, Islamabad, Pakistan
}

Correspondence should be addressed to Nayyar Mehmood; nayyarmaths@gmail.com

Received 10 April 2016; Revised 15 June 2016; Accepted 23 June 2016

Academic Editor: Adrian Petrusel

Copyright (C) 2016 N. Mehmood and A. Azam. This is an open access article distributed under the Creative Commons Attribution License, which permits unrestricted use, distribution, and reproduction in any medium, provided the original work is properly cited.

We discuss the existence of solution of a certain type of fuzzy partial differential inclusions with local conditions of integral types.

\section{Introduction}

The complexities of physical phenomena depict some kind of uncertainty, and dealing with uncertainty by fuzzy sets was introduced by Zadeh in [1]. For the betterment of mathematical modelling for physical problems, formulation by fuzzy differential equations plays an important role. The notion of fuzzy derivatives was introduced in [2]. In [3], the authors define these concepts by using Zadeh's extension principle, while, in [4], the fuzzy derivative was defined by generalized Hukuhara derivative. For more details about the theory of fuzzy differential equations, we refer the readers to the monograph [5].

The incomplete and uncertain systems can be modelled in a better way by virtue of fuzzy differential equations. The theory of fuzzy partial differential equations was initiated in [6]. After that many authors work out in this branch of analysis in a productive way, for example, [7-11] and the references therein. The importance of ordinary differential equations and partial differential equations in fuzzy sense is obvious. This field is in its growing age, and it interacts with many researchers. And up till now the literature is mainly concerned with fuzzy ordinary differential equations, inclusions, and fuzzy partial differential equations, but in this paper we present a model for hyperbolic type partial fuzzy differential inclusion with integral local conditions. In [12], the authors discuss the existence of solution of a fuzzy differential inclusion problem and, in [13], the authors extend the technique for a system of fuzzy differential inclusions. We extend this study to discuss the existence of solution of fuzzy partial differential inclusion. We also present an example to justify our main result.

\section{Preliminaries}

Let $K\left(\mathbb{R}^{n}\right)$ be the family of all nonempty, convex, and compact subsets of $\mathbb{R}^{n}$. The Hausdorff metric in $K\left(\mathbb{R}^{n}\right)$ is defined as follows:

$$
H(A, B)=\max \left\{\sup \inf _{a \in A}\|a-b\|, \sup _{b \in B}\|a-b\|\right\} .
$$

Then $\left(H, K\left(\mathbb{R}^{n}\right)\right)$ is a complete and separable space.

Definition 1 (see [13]). A fuzzy subset $A$ of $R^{n}$ is a function $A: R^{n} \rightarrow[0,1]$. The $\alpha$ open level and $\alpha$ closed level sets of $A$ are $(A)_{\alpha}$ and $[A]_{\alpha}$ and are defined as follows:

$$
\begin{array}{ll}
(A)_{\alpha}=\{x: A(x)>\alpha\} & \text { for } \alpha \in[0,1), \\
{[A]_{\alpha}=\{x: A(x) \geq \alpha\}} & \text { for } \alpha \in[0,1],
\end{array}
$$

respectively.

Definition 2 (see [13]). Denote by $E^{n}$ the set of all fuzzy sets of $R^{n}$, such that $u \in E^{n}$ satisfy the following properties:

(i) $u$ is normal; that is, there exists $x \in R^{n}$ such that $u(x)=1$.

(ii) $u$ is fuzzy convex; that is, for $x, y \in R^{n}$ and $\lambda \in[0,1]$, $u(\lambda x+(1-\lambda) x) \geq \min \{u(x), u(y)\}$. 
(iii) $u$ is upper semicontinuous; that is, for any $\alpha,[u]_{\alpha}$ is closed set.

(iv) $[u]_{0}=\overline{\left\{x \in R^{n}: u(x) \geq 0\right\}}$ is compact, where bar denotes the closure of the set.

It is obvious that, for each $u \in R^{n}$, the $\alpha$ closed level sets are compact and convex in $R^{n}$.

Lemma 3 (see [13]). If $u \in E^{n}$, then

(i) $[u]_{\alpha}$ is compact and convex for all $0 \leq \alpha \leq 1$,

(ii) for all $\alpha \leq \beta[u]_{\beta} \subset[u]_{\alpha}$,

(iii) if $\left\{\alpha_{n}\right\}$ is a nondecreasing sequence converging to some $\alpha$ in $[0,1]$, then $[u]_{\alpha}=\bigcap_{n \in \mathbb{N}}[u]_{\alpha_{n}}$.

If $\left\{A_{\alpha}: 0 \leq \alpha \leq 1\right\}$ is a family of subsets of $R^{n}$ satisfying the above conditions of the lemma, then there exists $u \in E^{n}$ such that $[u]_{\alpha}=A_{\alpha}$ and $[u]_{\alpha}=\overline{\bigcup_{0 \leq \alpha \leq 1} A_{\alpha}} \subset A_{0}$.

Definition 4 (see [12]). Let $(X, d)$ and $\left(Y, d_{1}\right)$ be two metric spaces and let $F: X \rightarrow 2^{Y}$ be a set-valued mapping; $F$ is said to be upper semicontinuous at $x_{0} \in X$ if and only if, for any neighborhood $U$ of $F\left(x_{0}\right)$, there exists a neighborhood $V$ of $x_{0}$, such that $T(x) \subset U$ for all $x \in V$.

Every fuzzy map $F: X \rightarrow E^{n}$ can generate a real valued function $\widetilde{F}: X \times R^{n} \rightarrow[0,1]$, where, for $x \in X, y \in R^{n}$, $\widetilde{F}(x, y)=F(x)(y):=F_{(x)(y)}$.

Definition 5 (see [13]). A fuzzy mapping $F: \Omega \rightarrow E^{n}$, where $\Omega \subset[0, a] \times[0, b] \times R^{n}$, is called lower open if $F_{(x, y, u)}(w)$ is lower semicontinuous in $(x, y, u) \in \Omega$.

\section{Main Results}

Proposition 6 (see [14]). Let D be a paracompact Hausdorff topological space, let $Y$ be a topological vector space, and let $F$ : $D \rightarrow 2^{Y}$ be a multivalued nonempty complex valued function. If $F$ has open lower sections, that is, for any $y \in Y, F^{-1}(y)$ is open in $D$, then there exists a continuous selection $f: D \rightarrow Y$ such that $f(x) \in F(x)$ for any $x \in D$.

Lemma 7 (see [15]). Let $\Omega \subset R \times R^{n} \times R^{m}$ be an open set and $(t, x, y) \in \Omega$ and $T: \Omega \rightarrow K\left(R^{n} \times R^{m}\right)$ an upper semicontinuous set-valued operator. Then there exists an open interval $J$ of $R$, for $a>0, M>0$, such that

(i) $J \times S_{a M}^{(x, y)} \subset \Omega$,

(ii) $\|T(t, x, y)\| \leq M$ on $J \times S_{a M}^{(x, y)}$.

Lemma 8 (see [15]). Let $X$ be a Banach space and let $S, T$ : $J \rightarrow K(X)$ be two multivalued measurable operators, where $K(X)$ is the collection of compact subsets of $X$. Then if $v(t) \epsilon$ $S(t)$ is measurable selection, then there exists a measurable selection $u(t) \in T(t)$ such that

$$
\|u(t)-v(t)\| \leq H(S(t), T(t))
$$

for all $t \in J$.
Problem 9. Let $\mathbb{R}^{n}$ be a Banach space with norm $\|\cdot\|$ and let $\Omega$ be an open subset of $[0, a] \times[0, b] \times \mathbb{R}^{n}$. Let $F$ : $\Omega \rightarrow F\left(\mathbb{R}^{n}\right)$ be a fuzzy mapping and $\alpha: \mathbb{R}^{n} \rightarrow[0,1)$ is an upper semicontinuous function. Consider the following partial fuzzy differential inclusion:

$$
\begin{gathered}
F_{(x, y, u(x, y))}\left(\frac{\partial^{2} u}{\partial x \partial y}+\frac{\partial\left(\xi_{1}(x, y) u(x, y)\right)}{\partial x}\right. \\
\left.+\frac{\partial\left(\xi_{2}(x, y) u(x, y)\right)}{\partial y}\right)>\alpha(u)
\end{gathered}
$$

for $(x, y, u) \in \Omega$, with local conditions of integral type

$$
\begin{aligned}
u(x, 0)+\int_{0}^{b} k_{1}(x) u(x, y) d y & =\zeta_{1}(x), \\
u(0,0)=0, & \\
u(0, y)+\int_{0}^{a} k_{2}(y) u(x, y) d x & =\zeta_{2}(y), \\
y & \in[0, b], x \in[0, a] .
\end{aligned}
$$

The problem is equivalent to

$$
\begin{aligned}
& \frac{\partial^{2} u}{\partial x \partial y}+\frac{\partial\left(\xi_{1}(x, y) u(x, y)\right)}{\partial x}+\frac{\partial\left(\xi_{2}(x, y) u(x, y)\right)}{\partial y} \\
& \quad \in\left(F_{(x, y, u(x, y))}\right)_{\alpha(u)}
\end{aligned}
$$

with integral type conditions $(C 1)$.

In the next theorem we prove the existence of solution of the above partial differential inclusion.

Theorem 10. Suppose that $F: \Omega \rightarrow F\left(\mathbb{R}^{n}\right)$ is bounded convex and lower open fuzzy surjection and $\alpha: \mathbb{R}^{n} \rightarrow[0,1)$ is an upper semicontinuous function such that $\left(F_{(x, y, u(x, y))}\right)_{\alpha(u(x, y))}$ is nonempty for each $(x, y, u) \in \Omega$. Then there exists $\tilde{h}: \Omega \rightarrow \mathbb{R}^{n}$ a continuous selection $\tilde{h}(x, y, u(x, y)) \in$ $\left(F_{(x, y, u(x, y))}\right)_{\alpha(u(x, y))}$ such that

$$
\begin{aligned}
& \frac{\partial^{2} u(x, y)}{\partial x \partial y}+\frac{\partial\left(\xi_{1}(x, y) u(x, y)\right)}{\partial x} \\
&+\frac{\partial\left(\xi_{2}(x, y) u(x, y)\right)}{\partial y}=\tilde{h}(x, y, u(x, y)) \\
& \forall(x, y, u) \in \Omega .
\end{aligned}
$$

Proof. We can define a set-valued function $\widetilde{F}: \Omega \rightarrow 2^{\mathbb{R}^{n}}$ as follows:

$$
\widetilde{F}(x, y, u(x, y))=\left(F_{(x, y, u(x, y))}\right)_{\alpha(u(x, y))},
$$


Clearly $\widetilde{F}(x, y, u(x, y))$ is nonempty for each $(x, y, u) \in \Omega$, and consider for $w, z \in \widetilde{F}(x, y, u(x, y))$ and $\theta \in[0,1]$

$$
\begin{aligned}
& F_{(x, y, u(x, y))}(\theta w+(1-\theta) z) \\
& \quad \geq \min \left\{F_{(x, y, u(x, y))}(w), F_{(x, y, u(x, y))}(z)\right\} \\
& \quad>\alpha(u(x, y))
\end{aligned}
$$

by convexity of $F$. Hence $\theta w+(1-\theta) z \in \widetilde{F}(x, y, u(x, y))$; thus $\widetilde{F}(x, y, u(x, y))$ is convex on $\Omega$.

Now we will show that $\widetilde{F}$ has open lower sections. For any $z \in R^{n}$

$$
\begin{aligned}
& \widetilde{F}^{-1}(z)=\{(x, y, u) \in \Omega: z \in \widetilde{F}(x, y, u(x, y))\} \\
& =\left\{(x, y, u) \in \Omega:\left(F_{(x, y, u(x, y))}\right)(z)>\alpha(u(x, y))\right\} .
\end{aligned}
$$

It is enough to prove that the complement of $\widetilde{F}^{-1}(z)$, that is, the set

$$
\begin{aligned}
& \left(\widetilde{F}^{-1}(z)\right)^{c} \\
& \quad=\left\{(x, y, u) \in \Omega:\left(F_{(x, y, u(x, y))}\right)(z) \leq \alpha(u(x, y))\right\},
\end{aligned}
$$

is closed. Let $\left(x_{n}, y_{n}, u_{n}\right)$ be a sequence in $\left(\widetilde{F}^{-1}(z)\right)^{c}$ such that $\left(x_{n}, y_{n}, u_{n}\right) \rightarrow(x, y, u)$. Since $F$ is lower open and $\alpha$ is upper semicontinuous we have

$$
F_{\left(x_{n}, y_{n}, u_{n}\right)}(z) \leq \alpha\left(u\left(x_{n}, y_{n}\right)\right),
$$

and we have

$$
\begin{aligned}
F_{(x, y, u)}(z) & \leq \lim _{n \rightarrow \infty} \inf F_{\left(x_{n}, y_{n}, u_{n}\right)}(z) \\
& \leq \lim _{n \rightarrow \infty} \sup \alpha\left(u\left(x_{n}, y_{n}\right)\right) \leq \alpha(u(x, y)) .
\end{aligned}
$$

Thus $(x, y, u) \in\left(\widetilde{F}^{-1}(z)\right)^{c}$ and hence $\widetilde{F}$ has open lower sections. Thus by Proposition 6 there exists a continuous selection $\widetilde{h} \in \widetilde{F}(x, y, u(x, y))$ for each $(x, y, u) \in \Omega$. As $F$ is a surjection and $\widetilde{F}(x, y, u(x, y))$ is bounded we get the problem

$$
\begin{array}{r}
\frac{\partial^{2} u(x, y)}{\partial x \partial y}+\frac{\partial\left(\xi_{1}(x, y) u(x, y)\right)}{\partial x} \\
+\frac{\partial\left(\xi_{2}(x, y) u(x, y)\right)}{\partial y}=\widetilde{h}(x, y, u(x, y)) \\
\forall(x, y, u) \in \Omega
\end{array}
$$

with local conditions $(C 1)$.

The following Tychonoff theorem will be used to prove the existence of solution of problem $(P 1)$ with local conditions $(C 1)$.

Theorem 11 (see [16] [Tychonoff]). Let E be a complete locally convex linear space and let $A$ be a closed and convex subset of E. Let $T: E \rightarrow E$ be continuous and $T(A) \subset A$. If $\overline{T(A)}$ is compact then $T$ has a fixed point.
Let $\mathbb{R}^{n}$ be an $n$-dimensional Euclidean space and let $I^{+}$be set of positive integers. Let

$$
\begin{aligned}
C & \left(\mathbb{R}^{n}, \mathbb{R}\right) \\
& =\left\{f: f \text { is continuous and } f: \mathbb{R}^{n} \longrightarrow \mathbb{R}\right\} .
\end{aligned}
$$

The topology on $C\left(\mathbb{R}^{2}, \mathbb{R}\right)$ is induced by families of seminorms

$$
\alpha_{k}^{\lambda_{k}}(\varphi)=\sup \left\{|\varphi(x, y)| e^{-\lambda_{k}(|x|+|y|)}: x, y \in \Omega\right\},
$$

where $\Omega$ is the bounded region in $\mathbb{R}^{2}, k \in I^{+}$, and $\lambda_{k} \geq 0$. It is well known that [17] this topology on $C\left(\mathbb{R}^{2}, \mathbb{R}\right)$ is complete and locally convex linear space. We use similar technique to that used in [18].

Observe that problem $(P 1)$ with $(C 1)$ is equivalent to finding fixed point of the following mapping $T: C\left(\mathbb{R}^{2}, \mathbb{R}\right) \rightarrow$ $C\left(\mathbb{R}^{2}, \mathbb{R}\right)$ defined by

$$
\begin{aligned}
(T u)(x, y)= & \zeta_{1}(x)+\zeta_{2}(y)-\int_{0}^{b} k_{1}(x) u(x, y) d y \\
& -\int_{0}^{a} k_{2}(y) u(x, y) d x \\
& -\int_{0}^{y} \xi_{1}(x, t) u(x, t) d t \\
& -\int_{0}^{x} \xi_{2}(s, y) u(s, y) d s \\
& +\int_{0}^{y} \xi_{1}(0, t) \zeta_{2}(t) d t \\
& +\int_{0}^{x} \xi_{2}(s, 0) \zeta_{1}(s) d s \\
& -\int_{0}^{x} \int_{0}^{b} \xi_{2}(s, 0) k_{1}(x) u(x, y) d s d y \\
& -\int_{0}^{y} \int_{0}^{a} \xi_{1}(0, t) k_{2}(y) u(x, y) d x d t \\
& +\int_{0}^{x} \int_{0}^{y} \tilde{h}(s, t, u(s, t)) d s d t .
\end{aligned}
$$

Condition 1. Consider $\widetilde{h}(x, y, u) \in C\left(\mathbb{R}^{3}, \mathbb{R}\right)$ and $|\widetilde{h}(x, y, u)|$ $\leq \varphi(x, y,|u|), \varphi \in C\left(\mathbb{R}^{2} \times \mathbb{R}^{+}, \mathbb{R}^{+}\right)$, and $\varphi$ is subadditive in $|u|$, for all $x, y \in \Omega$.

Condition 2. Consider $\zeta_{1}, \zeta_{2}, k_{1}, k_{2} \in C(\mathbb{R}, \mathbb{R})$ and $\xi_{1}, \xi_{2} \in$ $C\left(\mathbb{R}^{2}, \mathbb{R}\right)$.

Theorem 12. If Conditions 1 and 2 hold then problem (P1) with local conditions $(C 1)$ has a solution in $C\left(\mathbb{R}^{2}, \mathbb{R}\right)$.

Proof. For existence of the solution we need to prove that $T$ has a fixed point in $C\left(\mathbb{R}^{2}, \mathbb{R}\right)$. Clearly $T$ is continuous and compact in the topology of $C\left(\mathbb{R}^{2}, \mathbb{R}\right)$. It is sufficient to 
4

Journal of Function Spaces

prove that, for any closed bounded and convex subset $A$ of $C\left(\mathbb{R}^{2}, \mathbb{R}\right)$, we have $T(A) \subset A$. For this, consider

$$
\begin{aligned}
(T v)(x, y)= & \zeta_{1}(x)+\zeta_{2}(y)-\int_{0}^{b} k_{1}(x) v(x, y) d y \\
& -\int_{0}^{a} k_{2}(y) v(x, y) d x \\
& -\int_{0}^{y} \xi_{1}(x, t) v(x, t) d t \\
& -\int_{0}^{x} \xi_{2}(s, y) v(s, y) d s \\
& +\int_{0}^{y} \xi_{1}(0, t) \zeta_{2}(t) d t \\
& +\int_{0}^{x} \xi_{2}(s, 0) \zeta_{1}(s) d s \\
& -\int_{0}^{x} \int_{0}^{b} \xi_{2}(s, 0) k_{1}(x) v(x, y) d s d y \\
& -\int_{0}^{y} \int_{0}^{a} \xi_{1}(0, t) k_{2}(y) v(x, y) d x d t \\
& +\int_{0}^{x} \int_{0}^{y} \tilde{h}(s, t, v(s, t)) d s d t .
\end{aligned}
$$

The above equation implies

$$
\begin{aligned}
|(T v)(x, y)| \leq & \left|\zeta_{1}(x)\right|+\left|\zeta_{2}(y)\right| \\
& +\left|\int_{0}^{y} \xi_{1}(0, t) \zeta_{2}(t) d t\right| \\
& +\left|\int_{0}^{x} \xi_{2}(s, 0) \zeta_{1}(s) d s\right| \\
& +\left|\int_{0}^{b} k_{1}(x) v(x, y) d y\right| \\
& +\left|\int_{0}^{a} k_{2}(y) v(x, y) d x\right| \\
& +\left|\int_{0}^{y} \xi_{1}(x, t) v(x, t) d t\right| \\
& +\left|\int_{0}^{x} \xi_{2}(s, y) v(s, y) d s\right| \\
& +\left|\int_{0}^{x} \int_{0}^{b} \xi_{2}(s, 0) k_{1}(x) v(x, y) d s d y\right| \\
& +\left|\int_{0}^{y} \int_{0}^{a} \xi_{1}(0, t) k_{2}(y) v(x, y) d x d t\right| \\
& +\left|\int_{0}^{x} \int_{0}^{y} \tilde{h}(s, t, v(s, t)) d s d t\right|
\end{aligned}
$$

The subadditivity of $\varphi$ implies the following inequality [18]:

$$
\varphi(x, y,|u|) \leq \varphi(x, y, 1)[1+|u|] ;
$$

using this inequality in the above inequality, we get

$$
\begin{aligned}
|(T v)(x, y)| \leq & \left|\zeta_{1}(x)\right|+\left|\zeta_{2}(y)\right| \\
& +\left|\int_{0}^{x} \xi_{2}(s, 0) \zeta_{1}(s) d s\right| \\
& +\left|\int_{0}^{y} \xi_{1}(0, t) \zeta_{2}(t) d t\right| \\
& +\left|\int_{0}^{b} k_{1}(x) v(x, y) d y\right| \\
& +\left|\int_{0}^{a} k_{2}(y) v(x, y) d x\right| \\
& +\left|\int_{0}^{y} \xi_{1}(x, t) v(x, t) d t\right| \\
& +\left|\int_{0}^{x} \xi_{2}(s, y) v(s, y) d s\right| \\
& +\left|\int_{0}^{x} \int_{0}^{b} \xi_{2}(s, 0) k_{1}(x) v(x, y) d s d y\right| \\
& +\left|\int_{0}^{y} \int_{0}^{a} \xi_{1}(0, t) k_{2}(y) v(x, y) d x d t\right| \\
& +\left|\int_{0}^{x} \int_{0}^{y} \varphi(s, t, 1)[1+v(s, t)] d s d t\right|
\end{aligned}
$$

Let

$$
\begin{aligned}
M & =\max \left\{\left(\int_{0}^{b}\left|k_{1}(x)\right|^{2} d y\right)^{1 / 2},\right. \\
& \left(\int_{0}^{a}\left|k_{2}(y)\right|^{2} d y\right)^{1 / 2},\left(\int_{0}^{y}\left|\xi_{1}(x, t)\right|^{2} d t\right)^{1 / 2}, \\
& \left(\int_{0}^{x}\left|\xi_{2}(s, y)\right|^{2} d s\right)^{1 / 2}, \\
& \left.\left(\int_{0}^{b}\left|\xi_{1}(0, t) k_{2}(y)\right|^{2} d x\right)^{1 / 2}\right\} \\
K & =\left\{\left(\int_{0}^{b}|v(x, y)|^{2} d y\right)^{1 / 2}+\left(\int_{0}^{a}|v(x, y)|^{2} d y\right)^{1 / 2}\right. \\
& +\left(\int_{0}^{y}|v(x, t)|^{2} d t\right)^{1 / 2}+\left(\int_{0}^{x}|v(s, y)|^{2} d s\right)^{1 / 2} \\
& +\int_{0}^{b}\left(\int_{0}^{b}|v(x, y)|^{2} d y\right)^{1 / 2} d y \\
& \left.\left.+\int_{0}^{a}|v(x, y)|^{2} d y\right)^{1 / 2} d t\right\} .
\end{aligned}
$$


By applying Schwartz's inequality and using the above values of $M$ and $K$, we get

$$
\begin{aligned}
|(T) v(x, y)| \leq & \left|\zeta_{1}(x)\right|+\left|\zeta_{2}(y)\right| \\
& +\left|\int_{0}^{x} \xi_{2}(s, 0) \zeta_{1}(s) d s\right| \\
& +\left|\int_{0}^{y} \xi_{1}(0, t) \zeta_{2}(t) d t\right|+M K \\
& +\left|\int_{0}^{x} \int_{0}^{y} \varphi(s, t, 1) d s d t\right| \\
& +\left.\left.\left|\int_{0}^{x} \int_{0}^{y}\right| \varphi(s, t, 1)\right|^{2} d s d t\right|^{1 / 2} \\
& \left.\left.\cdot\left|\int_{0}^{x} \int_{0}^{y}\right| v(s, t)\right|^{2} d s d t\right|^{1 / 2} .
\end{aligned}
$$

Using the definition of given seminorm, we obtain

$$
\begin{aligned}
|(T) v(x, y)| \leq & \left|\zeta_{1}(x)\right|+\left|\zeta_{2}(y)\right| \\
& +\left|\int_{0}^{x} \xi_{2}(s, 0) \zeta_{1}(s) d s\right| \\
+ & \left|\int_{0}^{y} \xi_{1}(0, t) \zeta_{2}(t) d t\right| \\
+ & \left|\int_{0}^{x} \int_{0}^{y} \varphi(s, t, 1) d s d t\right|+\frac{6}{2 \lambda_{k}} \\
& \cdot M \alpha_{k}^{\lambda_{k}}(v) e^{\lambda_{k}(|x|+|y|)} \\
+ & \left.\left.\frac{1}{2 \lambda_{k}}\left|\int_{0}^{x} \int_{0}^{y}\right| \varphi(s, t, 1)\right|^{2} d s d t\right|^{1 / 2} \\
& \cdot \alpha_{k}^{\lambda_{k}}(v) e^{\lambda_{k}(|x|+|y|)} .
\end{aligned}
$$

Let

$$
\begin{aligned}
& B(x, y)=\max \left\{\left|\zeta_{1}(x)\right|+\left|\zeta_{2}(y)\right|\right. \\
& +\left|\int_{0}^{x} \xi_{2}(s, 0) \zeta_{1}(s) d s\right|+\left|\int_{0}^{y} \xi_{1}(0, t) \zeta_{2}(t) d t\right| \\
& +\left|\int_{0}^{x} \int_{0}^{y} \varphi(s, t, 1) d s d t\right|, 6 M, \\
& \left.\left.\left.\left|\int_{0}^{x} \int_{0}^{y}\right| \varphi(s, t, 1)\right|^{2} d s d t\right|^{1 / 2}\right\}
\end{aligned}
$$

using this we have

$$
\begin{aligned}
|(T) v(x, y)| \leq & B(x, y) \\
& +\frac{1}{\lambda_{k}} B(x, y) \alpha_{k}^{\lambda_{k}}(v) e^{\lambda_{k}(|x|+|y|)} ;
\end{aligned}
$$

this can be written as

$$
\alpha_{k}^{\lambda_{k}}(T v) \leq \alpha_{k}^{0}(B)+\frac{1}{\lambda_{k}} \alpha_{k}^{0}(B) \alpha_{k}^{\lambda_{k}}(v)
$$

by choosing $\lambda_{k}=2 \alpha_{k}^{0}(B)$ and $A=\left\{u \in C\left(\mathbb{R}^{2}, \mathbb{R}\right): \alpha_{k}^{\lambda_{k}}(u) \leq\right.$ $\left.2 \alpha_{k}^{0}(B)\right\}$, we have, for any $u \in A$,

$$
\alpha_{k}^{\lambda_{k}}(T u) \leq 2 \alpha_{k}^{0}(B)
$$

that is,

$$
T(A) \subset A \text {. }
$$

Hence, by Tychonoff Theorem, there exists $v \in A$ such that $T v=v$. This completes the theorem.

Problem 13. Now consider the following partial fuzzy differential inclusion:

$$
\begin{gathered}
F_{(x, y, u(x, y))}\left(\frac{\partial^{2} u}{\partial x \partial y}+\frac{\partial\left(\xi_{1}(x, y) u(x, y)\right)}{\partial x}\right. \\
\left.+\frac{\partial\left(\xi_{2}(x, y) u(x, y)\right)}{\partial y}\right) \geq \alpha(u),
\end{gathered}
$$

for $(x, y, u) \in \Omega$, with local conditions of the same integral type $(C 1)$.

The problem is equivalent to

$$
\begin{aligned}
& \frac{\partial^{2} u(x, y)}{\partial x \partial y}+\frac{\partial\left(\xi_{1}(x, y) u(x, y)\right)}{\partial x} \\
& \quad+\frac{\partial\left(\xi_{2}(x, y) u(x, y)\right)}{\partial y} \in\left[F_{(x, y, u(x, y))}\right]_{\alpha(u(x, y))}
\end{aligned}
$$

with integral local conditions (C1).

In the next theorem we find the existence result for a solution of above fuzzy partial differential inclusion.

Theorem 14. Let $F: \Omega \rightarrow E^{n}$ be $\alpha$-level uniformly continuous and fuzzy integrably bounded; $\alpha: \mathbb{R}^{n} \rightarrow[0,1]$ is uniformly continuous. If, for every $(x, y, u),(\bar{x}, \bar{y}, v)$ in $\Omega$, there exists $\delta \in$ $(0, a b)$ such that

$$
H(F(x, y, u), F(\bar{x}, \bar{y}, v)) \leq \frac{\delta}{(a b)^{2}}\|u-v\|
$$

then there exists a solution of Problem 13.

Proof. Define $\widetilde{F}: \Omega \rightarrow 2^{\mathbb{R}^{n}}$ by $\widetilde{F}(x, y, u)=[F(x, y, u)]_{\alpha(u(x, y))}$. We show that $\widetilde{F}(x, y, u)$ is upper semicontinuous. For a given $(\stackrel{\circ}{x}, \dot{y}, \stackrel{i}{u}) \in \Omega$, we can write the neighborhood of $\widetilde{F}(\dot{x}, \dot{y}, \stackrel{\circ}{u})$ as follows:

$$
S_{r}^{\widetilde{F}(\stackrel{\circ}{x}, \stackrel{\circ}{u})}=\left\{w \in \mathbb{R}^{n}: d(w, \widetilde{F}(\dot{x}, \dot{y}, \stackrel{\circ}{u}))<r\right\} .
$$


For $(x, y, u) \in \Omega$ and $v \in \widetilde{F}(x, y, u)$, we have

$$
\begin{aligned}
& d(v, \widetilde{F}(\stackrel{\circ}{x}, \stackrel{\circ}{y}, \stackrel{\circ}{u})) \leq H(\widetilde{F}(x, y, u), \widetilde{F}(\stackrel{\circ}{x}, \stackrel{\dot{y}}{,} \stackrel{\circ}{u})) \\
& =H\left([F(x, y, u)]_{\alpha(u)},[F(\stackrel{\circ}{x}, \dot{y}, \stackrel{\circ}{u})]_{\alpha(\mathfrak{u})}\right) \\
& \leq H\left([F(\stackrel{\circ}{x}, \stackrel{\circ}{y}, \stackrel{\circ}{u})]_{\alpha(\stackrel{\circ}{u})},[F(x, y, u)]_{\alpha(\mathfrak{u})}\right) \\
& +H\left([F(x, y, u)]_{\alpha(\mathfrak{u})},[F(x, y, u)]_{\alpha(u)}\right) \\
& \leq H(\widetilde{F}(\stackrel{\circ}{x}, \dot{y}, \stackrel{\circ}{u}), \widetilde{F}(x, y, u)) \\
& +H\left([F(x, y, u)]_{\alpha(\mathfrak{u})},[F(x, y, u)]_{\alpha(u)}\right) .
\end{aligned}
$$

Since $[F(x, y, u)]_{\alpha(u)}$ is uniformly continuous for each $\alpha(u)$ and $\alpha$ is also uniformly continuous and, using the above inequality, we can find a small enough neighborhood $U$ of $(\stackrel{\circ}{x}, \dot{y}, \dot{u})$ in $\Omega$, such that for all $(x, y, v) \in U$ and $w \in \widetilde{F}(x, y, v)$

$$
d(w, \widetilde{F}(\stackrel{\circ}{x}, \dot{y}, \stackrel{i}{u}))<r
$$

thus

$$
\widetilde{F}(U) \subset S_{r}^{\widetilde{F}(\stackrel{\circ}{x}, \stackrel{\circ}{y}, \stackrel{\circ}{u})}
$$

This means that $\widetilde{F}(x, y, u)$ is upper semicontinuous. So we find, from Lemma 7 , that there exists a real constant $\theta>0$ such that

$$
\max _{(x, y, u) \in \Omega}\|\widetilde{F}(x, y, u)\| \leq \theta
$$

Let $X=\left\{u \in C\left(I, \mathbb{R}^{n}\right):\left\|u-u_{0}\right\| \leq \theta\right.$, for all $(x, y) \in I=$ $\left.[0, a] \times[0, b], u\left(x_{0}, y_{0}\right)=u_{0}\right\}$ with a metric $d_{X}: X \times X \rightarrow$ $\mathbb{R} \cup\{+\infty\}$ defined by

$$
d_{X}=\sup _{(x, y) \in I}\{\|u(x, y)-v(x, y)\|\}
$$

Then $\left(X, d_{X}\right)$ is a complete generalized metric space [13]. Define $T: X \rightarrow 2^{X}$ by

$$
\begin{aligned}
& T(u)=\left\{\bar{u}(x, y): \bar{u}(x, y) \in u_{0}(x, y)\right. \\
& \left.+\int_{x_{0}}^{x} \int_{y_{0}}^{y}[F(s, t, u(s, t))]_{\alpha(u(s, t))} d s d t \text { a.e } I\right\},
\end{aligned}
$$

where

$$
\begin{aligned}
u_{0}(x, y)= & \zeta_{1}(x)+\zeta_{2}(y)-\int_{0}^{b} k_{1}(x) u(x, y) d y \\
& -\int_{0}^{b} k_{1}(x) u(x, y) d y \\
& -\int_{0}^{y} \xi_{1}(x, t) u(x, t) d t \\
& -\int_{0}^{x} \xi_{2}(s, y) u(s, y) d s
\end{aligned}
$$

$$
\begin{aligned}
& +\int_{0}^{y} \xi_{1}(0, t) \zeta_{2}(t) d t \\
& +\int_{0}^{x} \xi_{2}(s, 0) \zeta_{1}(s) d s \\
& -\int_{0}^{x} \int_{0}^{b} \xi_{2}(s, 0) k_{1}(x) u(x, y) d s d y \\
& -\int_{0}^{y} \int_{0}^{a} \xi_{1}(0, t) k_{2}(y) u(x, y) d x d t
\end{aligned}
$$

Here $\int_{x_{0}}^{x} \int_{y_{0}}^{y}[F(s, t, u(s, t))]_{\alpha(u(s, t))} d s d t$ is multivalued double integral of Aumann [19], and we defined it here as follows:

$$
\begin{aligned}
& \int_{x_{0}}^{x} \int_{y_{0}}^{y} \widetilde{F}(s, t, u(s, t)) d s d t \\
& \quad=\int_{x_{0}}^{x} \int_{y_{0}}^{y}[F(s, t, u(s, t))]_{\alpha(u(s, t))} d s d t=\left\{u_{0}\right. \\
& \quad+\int_{x_{0}}^{x} \int_{y_{0}}^{y} f(s, t, u(s, t)) d s d t \mid f: \Omega \\
& \quad \longrightarrow \mathbb{R}^{n} \text { is measurable selection for }[F(s, t, \\
& \left.u(s, t))]_{\alpha}\right\},
\end{aligned}
$$

for each $\alpha \in(0,1]$.

Clearly $T(u) \neq \varphi$ for all $u \in X$. Since the operator $\widetilde{F}(x, y, u)=[F(x, y, u)]_{\alpha(u(x, y))}$ is upper semicontinuous with compact values, by well known selection theorem of Kuratowski-Ryll-Nardzewski [20], $\widetilde{F}(x, y, u)$ has measurable selection $f(x, y, u) \in[F(x, y, u)]_{\alpha(u(x, y))}$ for all $(x, y, u) \in I \times R^{n}$, and $f(x, y, u)$ is Lebesgue integrable [4].

Let $u(x, y)=u_{0}+\int_{x_{0}}^{x} \int_{y_{0}}^{y} f(s, t, u(s, t)) d s d t \in T(u)$; thus $T(u) \neq \varphi$.

Next we show that $T(u)$ is closed for all $u \in X$. Let $u_{n}$ be a sequence in $T(u)$ that converges to $\dot{u} \in X$. Since

$$
u_{n} \in u_{0}+\int_{x_{0}}^{x} \int_{y_{0}}^{y} \widetilde{F}(s, t, u(s, t)) d s d t
$$

and $u_{0}+\int_{x_{0}}^{x} \int_{y_{0}}^{y} \widetilde{F}(s, t, u(s, t)) d s d t$ is closed [19], we have $\stackrel{\circ}{\epsilon} \epsilon$ $T(u)$.

We claim that $T$ is multivalued contraction; for this let $u_{2} \in T\left(u_{1}\right)$, which implies that there exists $f\left(x, y, u_{1}(x, y)\right) \in$ $\widetilde{F}\left(x, y, u_{1}(x, y)\right)$ such that

$$
u_{2}(x, y)=u_{0}+\int_{x_{0}}^{x} \int_{y_{0}}^{y} f\left(s, t, u_{1}(s, t)\right) d s d t
$$


By virtue of Lemma 8 [21], there exists a measurable selection $f\left(x, y, u_{2}(x, y)\right) \in \widetilde{F}\left(x, y, u_{2}(x, y)\right)$ such that

$$
\begin{aligned}
& \left\|f\left(x, y, u_{2}(x, y)\right)-f\left(s, t, u_{1}(s, t)\right)\right\| \\
& \quad \leq H\left(\widetilde{F}\left(x, y, u_{1}(x, y)\right), \widetilde{F}\left(x, y, u_{2}(x, y)\right)\right) \\
& \quad=H\left(\left[F\left(x, y, u_{1}(x, y)\right)\right]_{u_{1}(x, y)},\right. \\
& \left.\quad\left[F\left(x, y, u_{2}(x, y)\right)\right]_{u_{2}(x, y)}\right) \leq H\left(F\left(x, y, u_{1}(x, y)\right),\right. \\
& \left.\quad F\left(x, y, u_{2}(x, y)\right)\right) .
\end{aligned}
$$

Let

$$
u_{3}(x, y)=u_{0}+\int_{x_{0}}^{x} \int_{y_{0}}^{y} f\left(s, t, u_{2}(s, t)\right) d s d t
$$

Now consider

$$
\begin{aligned}
\| u_{3} & (x, y)-u_{2}(x, y)\|=\| u_{0} \\
& +\int_{x_{0}}^{x} \int_{y_{0}}^{y} f\left(s, t, u_{2}(s, t)\right) d s d t-u_{0} \\
& -\int_{x_{0}}^{x} \int_{y_{0}}^{y} f\left(s, t, u_{1}(s, t)\right) d s d t \| \\
& =\| \int_{x_{0}}^{x} \int_{y_{0}}^{y} f\left(s, t, u_{2}(s, t)\right) d s d t \\
& -\int_{x_{0}}^{x} \int_{y_{0}}^{y} f\left(s, t, u_{1}(s, t)\right) d s d t \| \\
& \leq \int_{x_{0}}^{x} \int_{y_{0}}^{y} \| f\left(x, y, u_{2}(x, y)\right) \\
& -f\left(s, t, u_{1}(s, t)\right) \| d s d t \\
& \leq \frac{\delta}{a b} d\left(u_{1}(x, y), u_{2}(x, y)\right) . \\
& \leq \int_{x_{0}}^{x} \int_{y_{0}}^{y} H\left(F\left(x, y, u_{1}(x, y)\right),\right. \\
& \left.F\left(x, y, u_{2}(x, y)\right)\right) d s d t \\
& \leq \int_{x_{0}}^{x} \int_{y_{0}}^{y} \frac{\delta}{(a b)^{2}} d\left(u_{1}(x, y), u_{2}(x, y)\right) \int_{x_{0}}^{x} \int_{y_{0}}^{y} d s d t \\
& \delta(x, y)-u_{2}(x, y) \| d s d t
\end{aligned}
$$

Similarly we will get

$$
\left\|u_{2}(x, y)-u_{3}(x, y)\right\| \leq \frac{\delta}{a b} d\left(u_{1}(x, y), u_{2}(x, y)\right) .
$$

Thus we have

$$
H\left(T\left(u_{1}\right), T\left(u_{2}\right)\right) \leq \frac{\delta}{a b} d\left(u_{1}(x, y), u_{2}(x, y)\right)
$$

for all $u_{1}(x, y), u_{2}(x, y) \in X$.

Since $\delta / a b \in(0,1)$, by Nadler's Theorem [22], there exists a fixed point $v \in T v$. Thus

$$
\begin{aligned}
v(x, y)= & \zeta_{1}(x)+\zeta_{2}(y)-\int_{0}^{b} k_{1}(x) v(x, y) d y \\
& -\int_{0}^{a} k_{2}(y) v(x, y) d x \\
& -\int_{0}^{y} \xi_{1}(x, t) v(x, t) d t \\
& -\int_{0}^{x} \xi_{2}(s, y) v(s, y) d s \\
& +\int_{0}^{y} \xi_{1}(0, t) \zeta_{2}(t) d t \\
& +\int_{0}^{x} \xi_{2}(s, 0) \zeta_{1}(s) d s \\
& -\int_{0}^{x} \int_{0}^{b} \xi_{2}(s, 0) k_{1}(x) v(x, y) d s d y \\
& -\int_{0}^{y} \int_{0}^{a} \xi_{1}(0, t) k_{2}(y) v(x, y) d x d t \\
& +\int_{x_{0}}^{x} \int_{y_{0}}^{y} f(s, t, v(s, t)) d s d t .
\end{aligned}
$$

Example 15. Consider the hyperbolic PDI

$$
\begin{aligned}
& \frac{\partial u}{\partial x \partial y}+\frac{\partial(x u)}{\partial x}+\frac{\partial(y u)}{\partial y} \\
& \quad \in\left[\chi_{\{(x+y)(\sin (x+y)+\cos (x+y))+\sin (x+y)\}}\right]_{\alpha(u)},
\end{aligned}
$$

with boundary conditions

$$
\begin{aligned}
& u(x, 0)=\sin (x), \\
& u(0, y)=\sin (y)
\end{aligned}
$$

and $x, y \in R$, where $R$ is a bounded rectangle in plane. Suppose that $F: \Omega \rightarrow F\left(\mathbb{R}^{n}\right)$ is bounded convex and lower open fuzzy surjection and $\alpha: \mathbb{R}^{n} \rightarrow[0,1)$ is an upper semicontinuous function such that

$$
\begin{aligned}
& {\left[F_{(x, y, u(x, y))}\right]_{\alpha(u(x, y))}} \\
& \quad:=\left[\chi_{\{(x+y)(\sin (x+y)+\cos (x+y))+\sin (x+y)\}}\right]_{\alpha(u(x, y))}
\end{aligned}
$$

is nonempty for each $(x, y, u) \in \Omega$, where $\chi$ is the characteristic function, as

$$
\begin{aligned}
& H\left(\left[\chi_{\{(x+y)(\sin (x+y)+\cos (x+y))+\sin (x+y)\}}\right]_{\alpha(u(x, y))},\right. \\
& \left.\quad\left[\chi_{\{(x+y)(\sin (x+y)+\cos (x+y))+\sin (x+y)\}}\right]_{\alpha(v(x, y))}\right)=0 .
\end{aligned}
$$


Then, by Theorem 10, there exists a continuous selection $f(x$, $y, u) \in\left[F_{(x, y, u(x, y))}\right]_{\alpha(u(x, y))}$ for each $(x, y, u) \in \Omega$, such that

$$
\begin{aligned}
& (x+y)(\sin (x+y)+\cos (x+y))+\sin (x+y) \\
& =f(x, y, u)
\end{aligned}
$$

which implies

$$
\begin{aligned}
(x & +y)(\sin (x+y)+\cos (x+y))+\sin (x+y) \\
& =\frac{\partial u}{\partial x \partial y}+\frac{\partial(x u)}{\partial x}+\frac{\partial(y u)}{\partial y} .
\end{aligned}
$$

Hence the solution of $\partial u / \partial x \partial y+\partial(x u) / \partial x+\partial(y u) / \partial y-(x+$ $y)(\sin (x+y)+\cos (x+y))-\sin (x+y)=0$ is thus

$$
u(x, y)=\sin (x+y) \text {. }
$$

\section{Competing Interests}

The authors have declared that they have no competing interests.

\section{References}

[1] L. A. Zadeh, "Fuzzy sets," Information and Computation, vol. 8, no. 3, pp. 338-353, 1965.

[2] S. S. L. Chang and L. A. Zadeh, "On fuzzy mapping and control," IEEE Transactions on Systems, Man, and Cybernetics, vol. 2, no. 1, pp. 30-34, 1972.

[3] D. Dubois and H. Prade, "Towards fuzzy differential calculus part 3: differentiation," Fuzzy Sets and Systems, vol. 8, no. 3, pp. 225-233, 1982.

[4] S. Seikkala, "On the fuzzy initial value problem," Fuzzy Sets and Systems, vol. 24, no. 3, pp. 319-330, 1987.

[5] V. Lakshmikantham and R. N. Mohapatra, Theory of Fuzzy Differential Equations and Inclusions, CRC Press, New York, NY, USA, 2004

[6] J. J. Buckley and T. Feuring, "Introduction to fuzzy partial differential equations," Fuzzy Sets and Systems, vol. 105, no. 2, pp. 241-248, 1999.

[7] A. Arara and M. Benchohra, "Fuzzy solutions for boundary value problems with integral boundary conditions," Acta Mathematica Universitatis Comenianae, vol. 75, no. 1, pp. 119-126, 2006.

[8] A. Arara, M. Benchohra, S. K. Ntouyas, and A. Ouahab, "Fuzzy solutions for hyperbolic partial differential equations," International Journal of Applied Mathematical Sciences, vol. 2, no. 2, pp. 181-195, 2005.

[9] Y.-Y. Chen, Y.-T. Chang, and B.-S. Chen, "Fuzzy solutions to partial differential equations: adaptive approach," IEEE Transactions on Fuzzy Systems, vol. 17, no. 1, pp. 116-127, 2009.

[10] H. V. Long, N. T. K. Son, N. T. M. Ha, and L. H. Son, "The existence and uniqueness of fuzzy solutions for hyperbolic partial differential equations," Fuzzy Optimization and Decision Making, vol. 13, no. 4, pp. 435-462, 2014.

[11] M. Nikravesh, L. A. Zadeh, and V. Korotkikh, Eds., Fuzzy Partial Differential Equations and Relational Equations, vol. 142 of Studies in Fuzziness and Soft Computing, Springer, Berlin, Germany, 2004.
[12] Y. G. Zhu and L. Rao, "Differential inclusions for fuzzy maps," Fuzzy Sets and Systems, vol. 112, no. 2, pp. 257-261, 2000.

[13] C. Min, Z.-B. Liu, L.-H. Zhang, and N.-J. Huang, "On a system of fuzzy differential inclusions," Filomat, vol. 29, no. 6, pp. 12311244, 2015.

[14] N. C. Yannelis and N. D. Prabhakar, "Existence of maximal elements and equilibria in linear topological spaces," Journal of Mathematical Economics, vol. 12, no. 3, pp. 233-245, 1983.

[15] J. P. Aubin and A. Cellina, Differential Inclusions: Set-Valued Maps and Viability Theory, vol. 264, Springer Science \& Business Media, Berlin, Germany, 2012.

[16] A. Tychonoff, "Ein fixpunktsatz," Mathematische Annalen, vol. 111, no. 1, pp. 767-776, 1935.

[17] M. A. Naimark, Normed Rings, P. Noordhoff, Groningen, The Netherlands, 1964.

[18] A. K. Aziz and J. P. Maloney, "An application of Tychonoff's fixed point theorem to hyperbolic partial differential equations," Mathematische Annalen, vol. 162, no. 1, pp. 77-82, 1965.

[19] R. J. Aumann, "Integrals of set-valued functions," Journal of Mathematical Analysis and Applications, vol. 12, pp. 1-12, 1965.

[20] K. Kuratowski and C. Ryll-Nardzewski, "A general theorem on selectors," Bulletin of the Polish Academy of Sciences, vol. 12, pp. 397-403, 1965.

[21] S. S. Chang, Fixed Point Theory with Applications, Chongqing Publishing House, Chongqing, China, 1984.

[22] J. Nadler, "Multi-valued contraction mappings," Pacific Journal of Mathematics, vol. 30, pp. 475-488, 1969. 


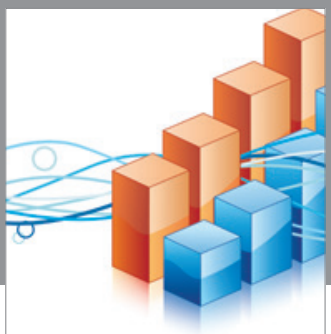

Advances in

Operations Research

vatem alat4

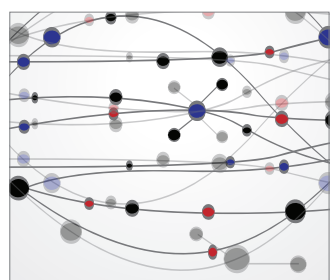

\section{The Scientific} World Journal
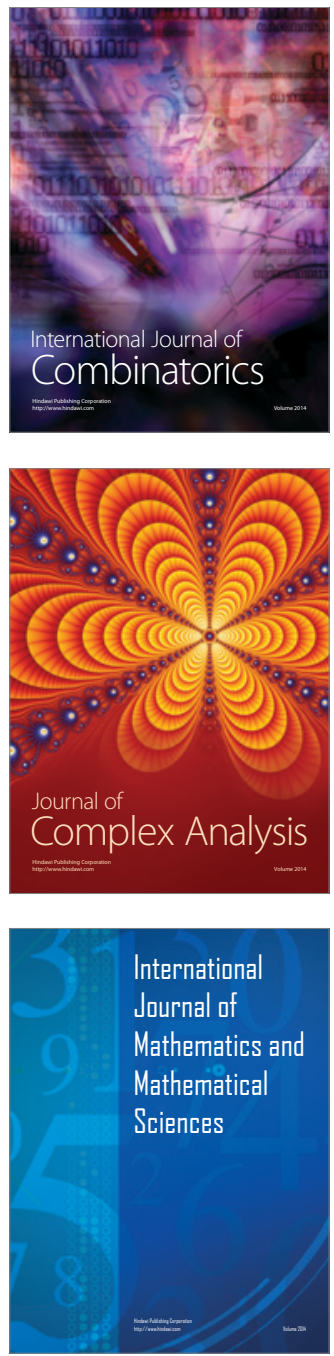
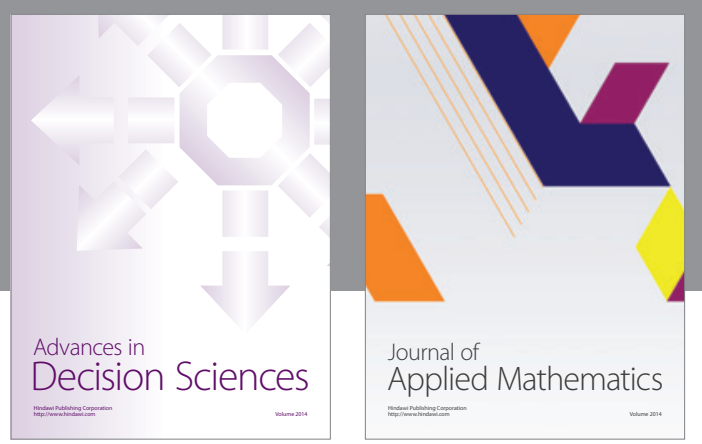

Algebra

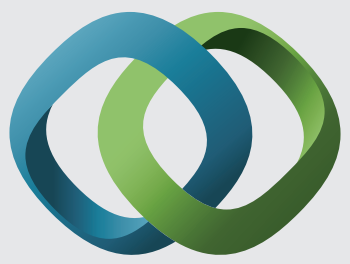

\section{Hindawi}

Submit your manuscripts at

http://www.hindawi.com
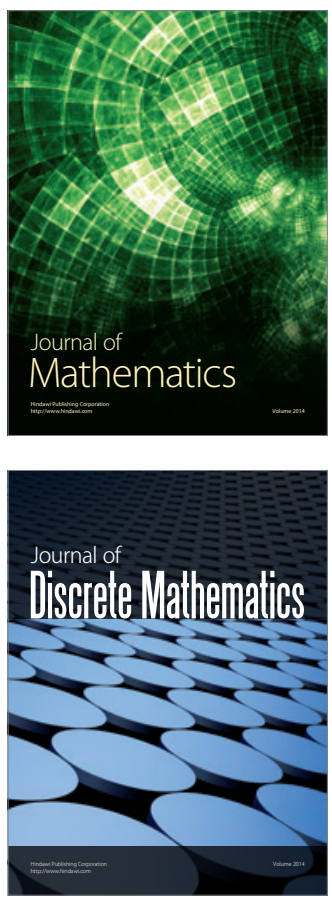

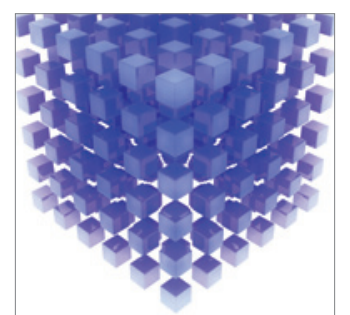

Mathematical Problems in Engineering
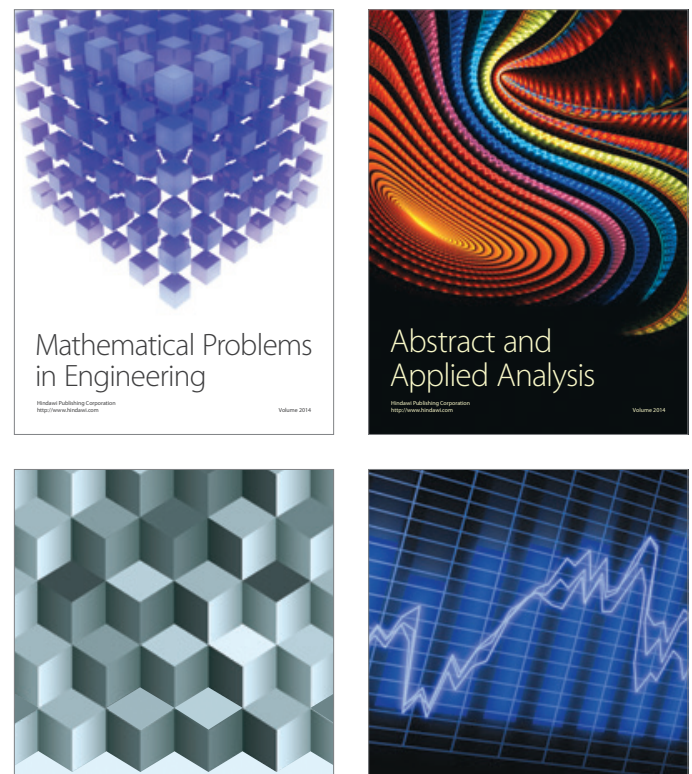

Journal of

Function Spaces

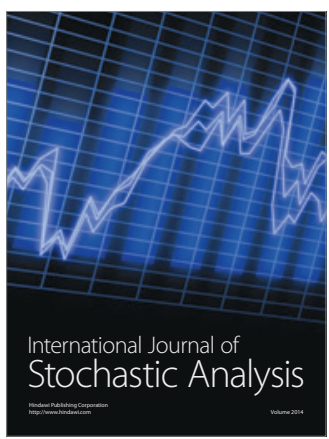

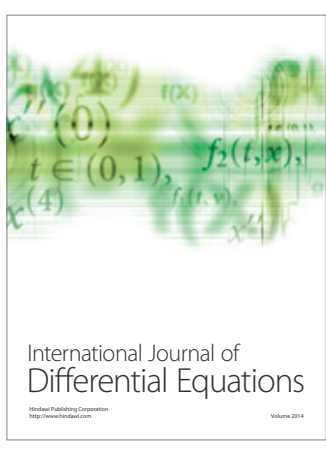
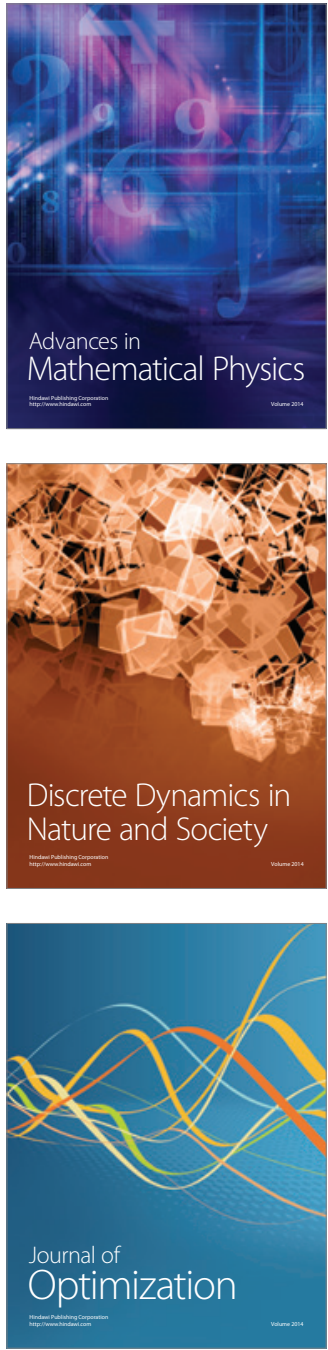